\title{
Attentional preparation based on temporal expectancy modulates processing at the perceptual level
}

\author{
ÁNGEL CORREA, JUAN LUPIÁÑEZ, and PÍO TUDELA \\ Universidad de Granada, Granada, Spain
}

\begin{abstract}
Research that uses simple response time tasks and neuroimaging has emphasized that attentional preparation based on temporal expectancy modulates processing at motor levels. A novel approach was taken to study whether the temporal orienting of attention can also modulate perceptual processing. A temporal-cuing paradigm was used together with a rapid serial visual presentation procedure, in order to maximize the processing demands of perceptual analysis. Signal detection theory was applied in order to examine whether temporal orienting affects processes related to perceptual sensitivity or to response criterion (indexed by $d^{\prime}$ and beta measures, respectively). If temporal orienting implies perceptual preparation, we would expect to observe an increase in perceptual sensitivity $\left(d^{\prime}\right)$ when a target appeared at expected, rather than unexpected, time intervals. Indeed, our behavioral results opened the possibility that focusing attention on time intervals not only enhances motor processing, as has been shown by previous research, but also might improve perceptual processing.
\end{abstract}

The environment continuously provides spatial and temporal information, which can be used to generate expectancies about when and where a relevant event will occur. Thus, expectancy helps us to develop a state of preparation in advance of the occurrence of stimuli, so that attention can be focused on the specific locations and moments at which stimuli are expected to occur. In this way, intelligent animals have some ability to predict the future - that is, to anticipate where and when an event will occur.

Attentional preparation based on expectancy generally implies two processes. First, sensory receptors and attention can be oriented to the expected place and moment, which improves stimulus perception. This improvement consists of an increase in the speed and/or accuracy of perceiving such stimuli. Moreover, preparation allows the anticipation of reactions to stimuli, in order to trigger a response as soon as they occur.

Concerning spatial attention, studies in which Posner's costs and benefits paradigm (Posner, Snyder, \& Davidson, 1980) has been used have shown that spatial expectancy influences processing. This attentional modulation can be deduced from faster and/or more accurate

This research was financially supported by the Spanish Ministerio de Educación y Cultura with Predoctoral Grant FPU-AP2000-3167 to the first author and by Research Grants MCyT, BSO2000-1503 and BSO2002-04308-C02-02 to J.L. and MCyT, BSO2000-1411-C02 to P.T. We thank Jeff Rouder and two anonymous reviewers for their helpful comments on the manuscript. Correspondence concerning this article should be addressed to Á. Correa or J. Lupiáñez, Departamento de Psicología Experimental y Fisiología del Comportamiento, Facultad de Psicología, Campus Universitario de Cartuja s/n, 18071 Granada, Spain (e-mail: act@ugr.es or jlupiane@ugr.es). responses and from amplification of visual evoked potentials for targets appearing at expected locations (see Mangun, 1995, for a review). Hence, such results are often interpreted in terms of a perceptual preparation based on spatial expectancy.

Concerning temporal attention, it has also been found that response times (RTs) are shorter when there is a match between the temporal expectancy for a target (early/ late) and the time interval (short/long) at which the target actually appears, than when they mismatch (see Nobre, 2001 , for a review). This so-called temporal-orienting effect (Correa, Lupiáñez, Milliken, \& Tudela, 2004) has reliably been found in detection tasks in which a temporalcuing paradigm has been used.

At present, however, it still remains unclear whether temporal-orienting effects are produced by perceptual preparation, motor preparation, or both. Indeed, this question cannot be addressed appropriately with behavioral data from classic RT experiments, since perceptual and motor preparation have the same effect-that is, to decrease RT. On the other hand, studies in which physiological measures have been used with detection tasks have supported the motor account of preparation (Coull, Frith, Büchel, \& Nobre, 2000; Coull \& Nobre, 1998; Griffin, Miniussi, \& Nobre, 2002; Miniussi, Wilding, Coull, \& Nobre, 1999).

However, it could be argued that the cerebral activation patterns observed in temporal-orienting studies might be partially due to the specific demands of the task used. Thus, motor areas may be involved mainly in detection tasks, whereas perceptual areas may be more involved in other tasks, such as discrimination. Discrimination tasks demand a more detailed perceptual analysis than detection tasks do, since processing of idiosyncratic features 
of the target is required. In contrast, detection tasks demand only a speeded response as soon as the target onset is detected, regardless of its visual features.

Thus, some of us have conducted several RT experiments in which a discrimination task was used instead of a detection task (Correa et al., 2004; Milliken, Lupiáñez, Roberts, \& Stevanovski, 2003). However, the finding that temporal expectancy facilitated stimulus discrimination is insufficient to support the hypothesis that temporal orienting produces perceptual preparation, since it could be argued that facilitation in discrimination tasks is produced by motor preparation of the two possible responses (Nobre, 2001).

In order to overcome this problem, we designed the present behavioral experiment, in which a good perceptual analysis of the target was essential to accomplishing the task. We were interested in conditions that make perception of the target difficult, in which accuracy is more important than the speed of the participant's responses, in order to discard motor preparation influences (see also Nobre, 2001). It was assumed that, if stimulus perception was improved by temporal expectancy in these difficult perceptual conditions without time pressure, this would suggest that temporal attention enhances perceptual processing.

How can such conditions in which perception is difficult be achieved? One possibility is to mask the target, so that its percept cannot be completely built by the visual system. Consequently, a rapid serial visual presentation (RSVP) procedure was used. This procedure consists of a very quick succession of stimuli presented at the same location on the screen. Given that stimuli occur very quickly, they mask each other, and perception of the target becomes quite difficult.

However, it makes sense to expect that it is possible to override some masking effects if attention is focused on the target (Enns \& Di Lollo, 1997). Specifically, this strategy was encouraged through cuing the likely time of occurrence. Given that the RSVP procedure does not allow attention to be directed to each item individually, a temporal cue would improve performance by indicating the best temporal window in which to search for the target.

If temporal orienting exclusively implied motor preparation, we would not expect to observe temporal-orienting effects in accuracy measures, since preparation for a speeded response is not relevant for this task. However, if temporal orienting implied perceptual preparation, we would expect to observe temporal-orienting effects in accuracy measures, since preparation for an enhanced perception of the target is essential for this task.

Signal detection theory (SDT) was applied to analyze the data. SDT provides two orthogonal indices that determine the observer's dichotomic decisions about a perceptual event under conditions of high uncertainty. The $d^{\prime}$ index is related to perceptual sensitivity, whereas the beta index is related to response criterion (Green \& Swets, 1966). High values of $d^{\prime}$ mean high perceptual sensitivity. High values of beta mean a strict criterion, whereas low values of beta mean a lax criterion. Thus, the following predictions are based on the assumption that $d^{\prime}$ is a reliable index of perceptual sensitivity.

If temporal orienting enhances perceptual processing, we should expect perceptual sensitivity $\left(d^{\prime}\right)$ to be affected by temporal expectancies - in particular, higher $d$ 's for targets appearing at validly, rather than at invalidly, cued time intervals.

Furthermore, an extra manipulation was incorporated. One group of participants was instructed to identify the letter X in the RSVP stream (single-target task), whereas the participants in the other group were instructed to identify either an X or an $\mathrm{O}$ (double-target task). Previous research carried out in our laboratory (Correa et al., 2004) has shown that when temporal expectancy is manipulated on a trial-by-trial basis, as in the present experiment, temporal-orienting effects were larger in simpleRT (detection) than in choice-RT (discrimination) tasks. Such differences were attributed to the difficulty of generating a temporal expectancy while maintaining in working memory the more complex task-set for the discrimination task (i.e., an arbitrary mapping between stimuli and responses). Thereby, we have proposed that the online generation of temporal expectancy requires controlled processing, which can be impaired as other demands on central resources increase.

In the present experiments, we further tested this hypothesis by manipulating the task set demands while holding constant the sensory input for both tasks. Assuming that the identification task was less demanding for a single target than for double targets, given that only one versus two perceptual templates had to be maintained in working memory, we expected to observe larger temporal-orienting effects in the former task.

\section{GENERAL METHOD}

\section{Participants}

One hundred twenty students of psychology from the University of Granada participated voluntarily. They were divided into two groups (Experiment 1) and four groups (Experiment 2) of 20 participants each. ${ }^{1}$ The participants were randomly assigned to the groups and performed the task in a room equipped with 15 computers.

\section{Stimuli}

MEL software (Schneider, 1988) controlled the experiment. All the stimuli appeared in the center of a 15 -in. monitor. They were a temporal cue, a set of distractors, and a target. The temporal cue was a red rectangle, $4 \mathrm{~mm}$ in height $\left(0.38^{\circ}\right.$ of visual angle). Its width was either 10 or $22 \mathrm{~mm}\left(0.95^{\circ}\right.$ or $\left.2.1^{\circ}\right)$. The distractors were a set of 24 letters of a standard keyboard, excluding the targets. The target was the letter $\mathrm{X}$ for the single-target group and either the letter $\mathrm{O}$ or the letter $\mathrm{X}$ for the double-target group. The distractors and the target were $24 \times 24$ pixels $\left(0.95^{\circ} \times 0.95^{\circ}\right)$ in size. In the single-target group, the participants pressed one key $(Z$ or $M)$ if the target $(\mathrm{X})$ was presented and the other if the target was absent. In the doubletarget group, the participants pressed one of the same two keys, depending on whether the $\mathrm{X}$ target or the $\mathrm{O}$ target was presented. The assignments were counterbalanced across participants.

\section{Procedure}

The participants sat approximately $60 \mathrm{~cm}$ in front of the screen. They were informed that the temporal cue would help them to pre- 
dict when the target would appear and were encouraged to respond as accurately as possible. However, they were not asked to make speeded responses.

The temporal cue appeared for $750 \mathrm{msec}$ and was followed by a fast stream of 43 letters (see Figure 1). Items were presented for $14 \mathrm{msec}$ each, and there was an interstimuli interval (ISI) of $28 \mathrm{msec}$ between them. In the first 9 positions of the stream, only distractors were presented. The target could appear, only once, in either Position 10 or 34, depending on stimulus onset asynchrony (SOA). Afterward, 9 extra distractors were presented. Distractors were randomly selected from the set of 24 .

Given that there was no time pressure, the participants responded after the stream had disappeared. The next trial started after a response.

There were six blocks of 60 trials (the first one considered to be practice) with a rest between them. Each set of 30 target trials consisted of 20 valid trials and 10 invalid trials (validity proportion of .66). The cue indicated that the target was likely to appear early on one half of trials and late on the remaining half. With early cues, the target appeared $414 \mathrm{msec}$ after cue onset (Position 10; short SOA) on two thirds of the trials (valid trials) and 1,057 msec after cue onset (Position 34; long SOA) on one third of the trials (invalid trials). With late cues, instead, the target appeared 1,057 msec after cue onset on two thirds of the trials and $414 \mathrm{msec}$ after cue onset on one third of the trials.

Importantly, the same procedure and sensory stimulation were used for the single-target and the double-target groups. The only difference was that the single-target group was given instructions to identify the $\mathrm{X}$ target (they were not informed about the $\mathrm{O}$, which appeared anytime the $\mathrm{X}$ was not presented), whereas the double-target group was instructed to identify the presence of either the $\mathrm{X}$ target or the $\mathrm{O}$ target.

\section{Design}

Experiments 1 and 2 had a 2 (task: single-target or double-target) $\times$ 2 (cue validity: valid or invalid) $\times 2$ (SOA: 414 or $1,057 \mathrm{msec}$ ) mixed factor design. Task was manipulated between subjects. Cue validity and SOA were manipulated within subjects and were randomly intermixed within blocks. Indices of perceptual sensitivity $\left(d^{\prime}\right)$ and response criterion (beta) were the dependent variables. For computing $d^{\prime}$ and beta similarly for the single-target and the double-target groups, the target was defined as $\mathrm{X}$ for the two groups. Thus, a yes (or $X$ ) response to an $\mathrm{X}$-present trial was considered a hit, whereas a yes (or $X$ ) response to an $\mathrm{X}$-absent trial (i.e., O present) was considered a false alarm.

\section{RESULTS}

\section{Experiment 1}

Mean $d^{\prime}$ s, betas, hit rates, and false alarm rates for each experimental condition in Experiments 1 and 2 are presented in Table 1.

Mean $d^{\prime}$ s and betas were submitted to separate mixed factor analyses of variance (ANOVAs) with cue validity and SOA as within-subjects variables and task as a between-subjects variable (this same analysis was used in Experiment 2).

$\boldsymbol{d}^{\prime}$ analysis. The main effect of $\operatorname{task}[F(1,38)=11.61$, $p<.002]$ showed that $d^{\prime}$ s were higher in the single-target task than in the double-target task. More interesting, there was a main effect of cue validity $[F(1,38)=7.33$, $p<.01]$, so that $d^{\prime}$ 's were higher for validly than for in-

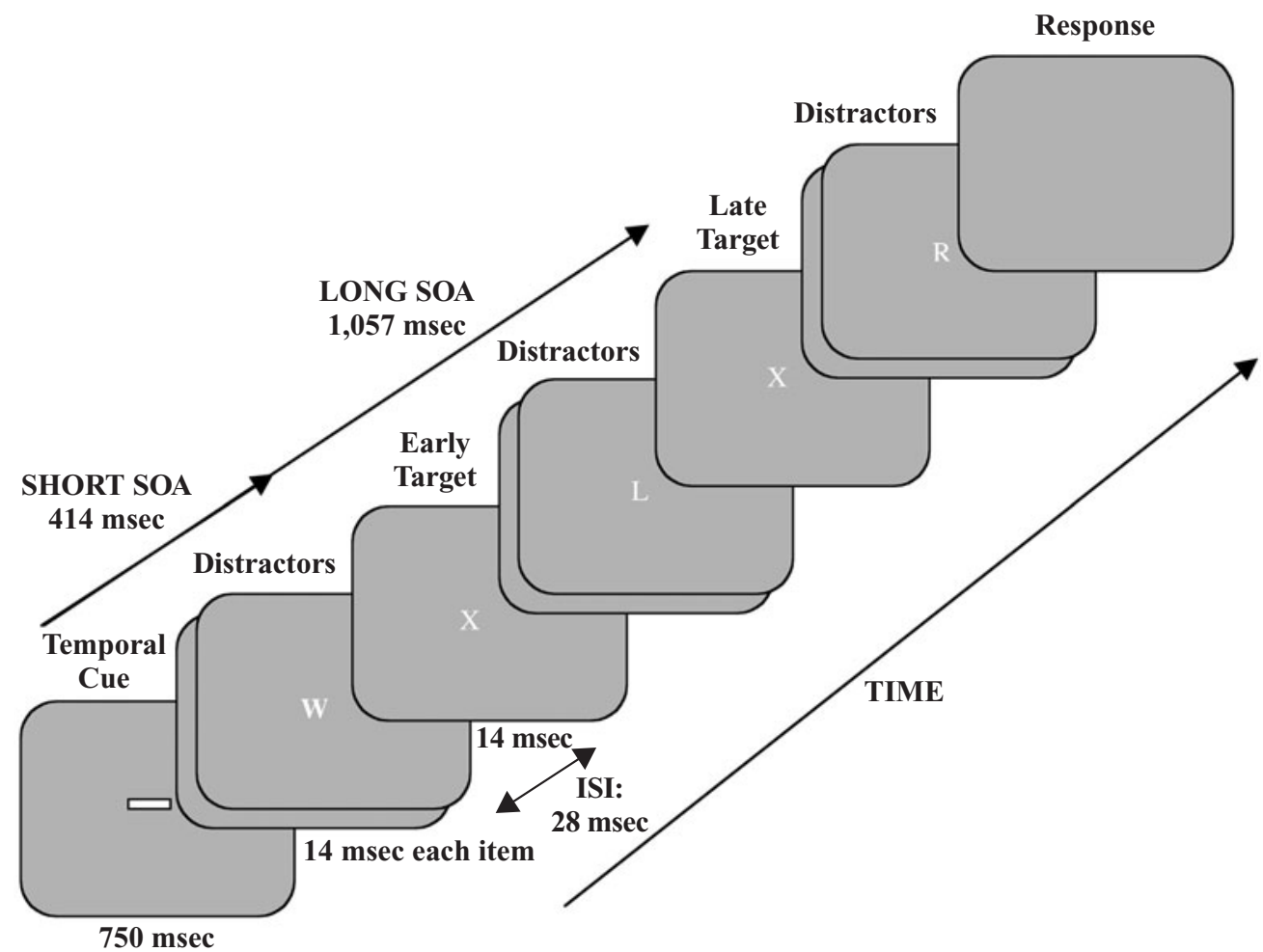

Figure 1. Sequence of events on a trial. 
Table 1

Mean $d$ 's, Betas, Hit Rates, and False Alarm Rates for Each Experimental Condition in Experiments 1 and 2

\begin{tabular}{|c|c|c|c|c|c|c|c|c|c|c|}
\hline \multirow[b]{3}{*}{ Experiment } & \multirow[b]{3}{*}{ Task } & \multirow[b]{3}{*}{ Cue } & \multicolumn{8}{|c|}{ Stimulus Onset Asynchrony } \\
\hline & & & \multicolumn{4}{|c|}{ Short (414 msec) } & \multicolumn{4}{|c|}{ Long $(1,057 \mathrm{msec})$} \\
\hline & & & $d^{\prime}$ & Beta & Hits & False Alarms & $d^{\prime}$ & Beta & Hits & False Alarms \\
\hline \multirow[t]{4}{*}{1} & Single target & Early & 0.63 & 1.24 & .53 & .31 & 0.44 & 1.16 & .51 & .35 \\
\hline & & Late & 0.52 & 1.16 & .47 & .29 & 0.82 & 1.38 & .57 & .28 \\
\hline & Double target & Early & 0.44 & 0.97 & .59 & .43 & 0.22 & 1.06 & .56 & .48 \\
\hline & & Late & 0.25 & 0.93 & .56 & .47 & 0.42 & 0.89 & .61 & .45 \\
\hline \multirow[t]{4}{*}{2} & Single target & Early & 0.91 & 1.51 & .58 & .28 & 0.58 & 2.41 & .44 & .28 \\
\hline & & Late & 0.45 & 2.05 & .37 & .25 & 1.05 & 2.04 & .56 & .22 \\
\hline & Double target & Early & 0.50 & 0.98 & .63 & .44 & 0.44 & 1.02 & .61 & .46 \\
\hline & & Late & 0.31 & 1.34 & .60 & .51 & 0.62 & 0.92 & .65 & .45 \\
\hline
\end{tabular}

Note-Hits and false alarms do not match to $d^{\prime}$ and beta in this table, since these are means computed over participants.

validly cued targets. Moreover, as can be seen in Figure 2 , this validity effect did not depend on a specific SOA, since the interaction between cue validity and SOA was far from significance $(p>.27)$.

Beta analysis. The main effect of task $[F(1,38)=$ $10.85, p<.002]$ revealed that betas were higher in the single-target task than in the double-target task. This effect was modulated by cue validity $[F(1,38)=5.61, p<$ $.02]$, so that the higher betas in the single-target task were observed for valid cues. Such an effect could also reflect a modulation of temporal attention on late processing stages (Miniussi et al., 1999), such as decision processes. Nevertheless, the main effect of cue validity was far from significance $(F<1)$.

\section{Experiment 2}

The primary aim of Experiment 2 was to replicate the most important finding in Experiment 1 - that is, an increase of perceptual sensitivity, presumably produced by the temporal orienting of attention. Moreover, we further explored the predicted interaction between task and cue validity, which was not observed in Experiment 1. Also, we focused on the unexpected main effect of task on $d^{\prime}$, since we did not have any predictions concerning the observed decrement in performance produced by the knowledge of the alternative category (i.e., the $\mathrm{O}$ target).

$\boldsymbol{d}^{\prime}$ analysis. The main effects of task and SOA were significant $[F(1,78)=13.98, p<.001$, and $F(1,78)=$ $5.38, p<.03$, respectively], so that $d^{\prime}$ s were higher in the

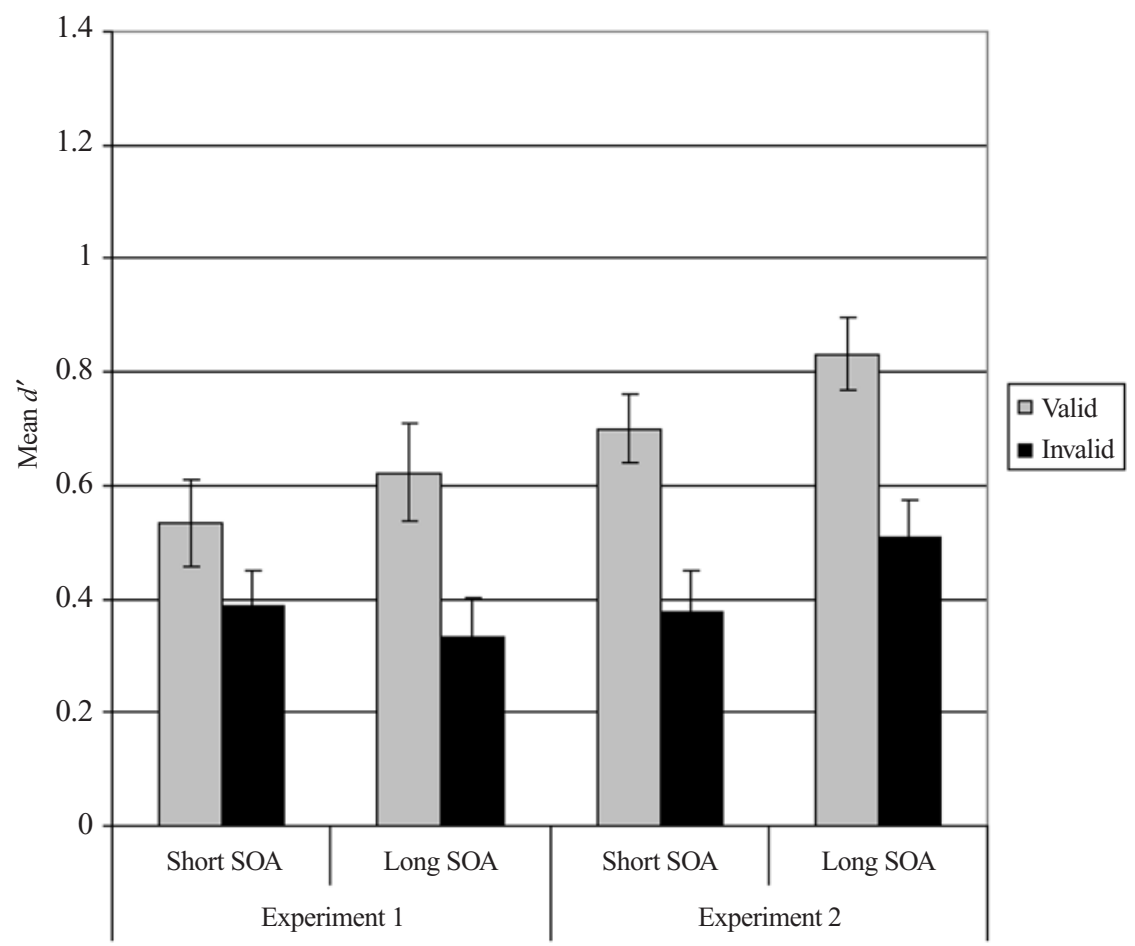

Figure 2. Mean $d^{\prime}$ s in Experiments 1 and 2, as a function of cue validity and stimulus onset synchrony (SOA). Note the validity effects for both short and long SOAs. 
single-target task than in the double-target task and $d^{\prime}$ s were higher at the long SOA than at the short SOA. The task effect replicated the results in Experiment 1-that is, having more information about the potential targets produced a decrease in performance. The SOA effect might be due to perceptual preparation processes not being completed by the time the target appeared at the shorter SOA.

Crucially, as was found in Experiment 1, there was a main effect of cue validity $[F(1,78)=21.10, p<.001]$. As is shown in Figure 2, significant validity effects were observed at both the short SOA $[F(1,78)=12.80, p<$ $.001]$ and the long SOA $[F(1,78)=13.56, p<.001]$.

This effect tended to be modulated by task $[F(1,78)=$ $3.83, p<.054]$. As was predicted, the temporal-orienting effect tended to be larger in the single-target task than in the double-target task.

Beta analysis. The only significant effect was the main effect of task $[F(1,78)=9.65, p<.003]$, which revealed that betas were higher in the single-target task than in the double-target task. This might be a consequence of different ways of responding to uncertainty about target occurrence. Uncertainty would trigger more no responses in the single-target task, whereas the yes and the no (i.e., $\mathrm{X}$ and $\mathrm{O}$ ) responses would be equal in the double-target task. Then, the $\mathrm{X}$ responses would be more frequent in the latter task, thus producing lower betas.

Thus, in contrast to $d^{\prime}$, beta was independent of the effect of cue validity in both experiments (except for the single-target group, Experiment 1). Therefore, the manipulation of cue validity produced changes mainly in perceptual sensitivity, but not in response criterion.

The effect of task on temporal orienting when the levels of performance are equated. It can be argued that the differential validity effects observed in the singleand the double-target groups could be due simply to differences in performance levels between the two tasks, rather than to differences in task set demands. According to this, we would expect similar validity effects for both tasks if performance levels are equated. In order to investigate this baseline confound, Experiment 2 was reanalyzed with performance included as a factor, so that we could explore its influence on validity effects and compare both tasks more reliably, once they had comparable levels of performance. Thus, each group of participants was divided into two subgroups, high versus low performance, by use of a median-split procedure on the participants' average $d^{\prime}$ across the four within-subjects conditions. Mean $d^{\prime}$ 's were then submitted to a mixed factor ANOVA with cue validity and SOA as within-subjects variables and performance (high/low) and task (singletarget/double-target) as between-subjects variables.

The analyses revealed significant main effects of performance, task, SOA, and cue validity $[F(1,76)=81.48$, $p<.001, F(1,76)=25.16, p<.001, F(1,76)=5.11$, $p<.03, F(1,76)=20.72, p<.001$, respectively]. It could not be otherwise: The low-performance group had a lower $d^{\prime}(0.38)$, than the high-performance group (0.85). The other main effects were as explained above.

Importantly, once the noise due to the between-subjects differences was reduced in this analysis (by including performance as a factor), the interaction between task and cue validity was significant $[F(1,76)=4.06, p<$ $.05]$, showing larger validity effects for the single-target task than for the double-target task, as we had predicted in accord with previous findings in our laboratory (Correa et al., 2004). And more relevant, this interaction was not modulated by performance $(F<1)$. As can be seen in Figure 3, the larger effects for the single-target task relative to the double target task are still present for both the low- and the high-performance groups. No other interactions were significant (all $p>.05$ ).

This result suggests that the effects of task on temporal orienting cannot be explained just in terms of differences in global performance. Although it is possible that dividing the participants ad hoc could have introduced other confounding selection effects, it is clear (see Figure 3 ) that the validity effect was greater for the singletarget task (low-performance group; mean $d^{\prime}=0.46$ ) than for the double-target task (high-performance group; mean $d^{\prime}=0.67$ ), despite the former having a worse overall performance.

\section{GENERAL DISCUSSION}

The present research provides new findings concerning the traditional debate about the specific influences of attention on processing. Such influences are better known in the space domain than in the time domain. Thus, it could be interesting to elucidate whether attentional preparation based on temporal expectancy modulates processing at the perceptual level or the motor level. Neuroimaging research is one approach by which to address this issue. However, the methodology used in the experiments reported here represents a novel approach based on behavioral measures, which has also provided useful information.

The following are the major results concerning our main measure $\left(d^{\prime}\right)$. First, our central hypothesis has been consistently supported by robust cue validity effects observed in $d^{\prime}$ in Experiments 1 and 2, so that valid temporal cues produced increments in the participants' perceptual sensitivity.

In relation to the effect of task, it was found in both experiments that performance in the single-target task was better than that in the double-target task. This finding could be surprising, considering that both tasks were identical in perceptual and motor terms. However, in the double-target group, more information was provided to find the target, as compared with the single-target group, so that the former knew that the target could be either an $\mathrm{X}$ or an $\mathrm{O}$, whereas the latter could search only for the $\mathrm{X}$ target. At first, one might expect that, the more information we have about what is being searched for, the higher the likelihood of finding it. However, this apparent 


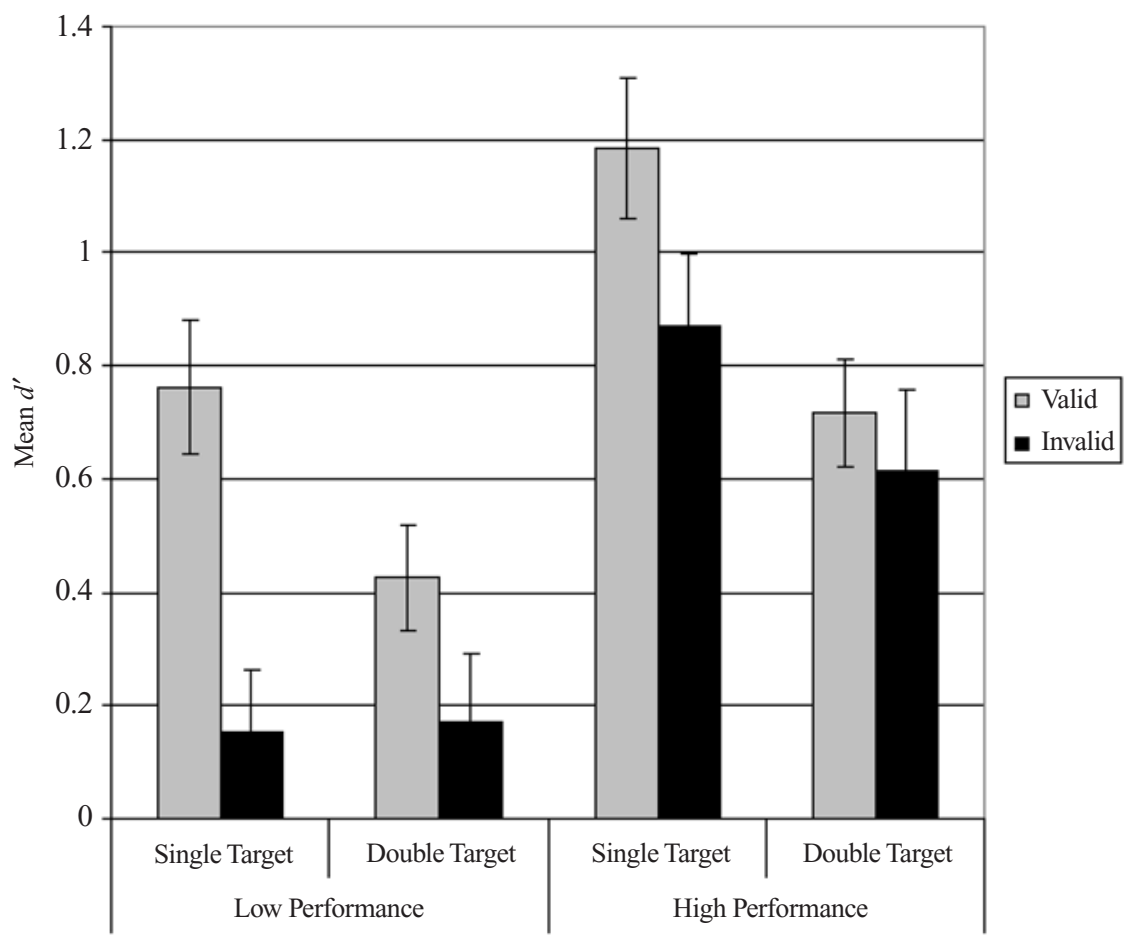

Figure 3. Mean $d^{\prime}$ 's in Experiment 2, as a function of cue validity, task, and performance (low performance [left panel] vs. high performance [right panel]). Note that the validity effects in the single-target task are larger than those in the double-target task.

paradox is easily explained from the memory search findings (Sternberg, 1966), since the search set size is higher for the double-target than for the single-target group.

In addition, such a feature that made harder the doubletarget task was also reflected in the interaction between task and cue validity. We assume that the double-target task was more demanding than the single-target task and, so, the generation of temporal expectancy was partly hindered by the former task. The present findings appear to be consistent with the conclusions drawn from previous research (Correa et al., 2004). In this sense, we suggest that on-line generation of temporal expectancy involves controlled processing (e.g., time estimation processes underlying temporal orienting). Hence, the ability to dynamically focus attention on time with success depends largely on other, simultaneous cognitive demands.

In summary, temporal-orienting effects have been consistently observed only in the measure related to perceptual sensitivity, which suggests that temporal attention can enhance early perceptual processing. However, there exists the possibility that our accuracy effects were produced by postperceptual factors instead. For example, an increase in sensitivity might be due to participants' encoding strategies of sampling more evidence (or preferentially rehearsing information) from the cued versus the uncued time interval. It is also possible, then, that sensory processes are equivalent at both cued and uncued intervals, whereas the attended information could have an earlier and privileged access to late decision processes (Hawkins et al., 1990).

Nevertheless, there is also important evidence suggesting that such effects can be reasonably related to perceptual processing. In particular, several visual attention studies have linked variations in $d^{\prime}$ both to modulations of sensory-evoked processing in the extrastriate visual cortex, as measured by ERPs (Luck et al., 1994), and to modulations of early visual cortex activity (V1, V2, and V3), as measured by event-related fMRI (Ress, Backus, \& Heeger, 2000). Furthermore, the paradigm used by Luck et al. was designed to prevent some of the confounds mentioned above.

Therefore, the findings obtained with this novel approach would challenge the notion that temporal orienting implies motor processes exclusively, rather than perceptual preparation, as has been reported by physiological studies (Griffin et al., 2002; Miniussi et al., 1999; but see Griffin et al., 2002, Experiment 1, for some evidence supporting perceptual enhancement). Previous studies have shown that directing attention to time intervals enhanced the speeded responses that are habitually demanded in simple RT tasks. Presumably, such results reflect mainly the contribution of temporal-orienting processes to motor preparation. Furthermore, we have found that focusing attention on a time interval could also enhance processes presumably related to perceptual sensitivity, which are 
essential to accomplishing the task used in this experiment. This finding is in line with a recent study that reported attentional modulations of sensory neurons from area V4 of the visual cortex in monkeys, as a consequence of an anticipation of the timing of relevant events (Ghose \& Maunsell, 2002). It makes sense, then, to expect that temporal attention, analogously to spatial attention, can flexibly enhance processing at different stages, according to the most relevant demands of the task at hand.

\section{REFERENCES}

Correa, Á., Lupiáñez, J., Milliken, B., \& Tudela, P. (2004). Endogenous temporal orienting of attention in detection and discrimination tasks. Perception \& Psychophysics, 66, 264-278.

Coull, J. T., Frith, C. D., Büchel, C., \& Nobre, A. C. (2000). Orienting attention in time: Behavioural and neuroanatomical distinction between exogenous and endogenous shifts. Neuropsychologia, 38, 808-819.

Coull, J. T., \& Nobre, A. C. (1998). Where and when to pay attention: The neural systems for directing attention to spatial locations and to time intervals as revealed by both PET and PMRI. Journal of Neuroscience, 18, 7426-7435.

ENNS, J. T., \& Di Lollo, V. (1997). Object substitution: A new form of masking in unattended visual locations. Psychological Science, 8, 135-139.

GHOse, G. M., \& Maunsell, J. H. R. (2002). Attentional modulation in visual cortex depends on task timing. Nature, 419, 616-620.

Green, D. M., \& Swets, J. A. (1966). Signal detection theory and psychophysics. New York: Wiley.

Griffin, I. C., Miniussi, C., \& Nobre, A. C. (2002). Multiple mechanisms of selective attention: Differential modulation of stimulus processing by attention to space of time. Neuropsychologia, 40, 2325-2340.

Hawkins, H. L., Hillyard, S. A., LuCK, S. J., Mouloua, M., DowNING, C. J., \& WoodWARD, D. P. (1990). Visual attention modulates signal detectability. Journal of Experimental Psychology: Human Perception \& Performance, 16, 802-811.
Luck, S. J., Hillyard, S. A., Mouloua, M., WoldorfF, M. G., Clark, V. P., \& Hawkins, H. L. (1994). Effects of spatial cuing on luminance detectability: Psychophysical and electrophysiological evidence for early selection. Journal of Experimental Psychology: Human Perception \& Performance, 20, 887-904.

MANGUN, G. R. (1995). Neural mechanisms of visual selective attention. Psychophysiology, 32, 4-18.

Milliken, B., Lupiáñez, J., Roberts, M., \& Stevanovski, B. (2003). Orienting in space and time: Joint contributions to exogenous spatial cuing effects. Psychonomic Bulletin \& Review, 10, 877-883.

Miniussi, C., Wilding, E. L., Coull, J. T., \& Nobre, A. C. (1999). Orienting attention in time: Modulation of brain potentials. Brain, 122, 1507-1518.

NoBRE, A. C. (2001). Orienting attention to instants in time. Neuropsychologia, 39, 1317-1328.

Posner, M. I., SNYDER, C. R. R., \& Davidson, B. J. (1980). Attention and the detection of signals. Journal of Experimental Psychology: General, 109, 160-174.

Ress, D., BACKUS, B. T., \& HeEger, D. J. (2000). Activity in primary visual cortex predicts performance in a visual detection task. Nature Neuroscience, 3, 940-945.

SCHNEIDER, W. (1988). Micro Experimental Laboratory: An integrated system for IBM PC compatibles. Behavior Research Methods, Instruments, \& Computers, 20, 206-217.

STERnBERG, S. (1966). High-speed scanning in human memory. Science, 153, 652-654.

\section{NOTES}

1. The four groups in Experiment 2 were collapsed into two: singletarget and double-target tasks. Originally, the $\mathrm{X}$ target frequency was manipulated in order to explore changes in response criterion as follows: two groups of .50 in Experiment 1 and two groups of .25 and .75, respectively, in Experiment 2. However, the detailed results are not reported here, since this manipulation was a secondary aim of the study and no clear results were obtained.

(Manuscript received December 12, 2003; revision accepted for publication June 5, 2004.) 